\title{
BER Performance of Free-Space Optical Transmission with Spatial Diversity
}

\author{
S. Mohammad Navidpour, Member, IEEE, Murat Uysal, Member, IEEE, and Mohsen Kavehrad, Fellow, IEEE
}

\begin{abstract}
Free space optical (FSO) communications is a cost-effective and high bandwidth access technique, which has been receiving growing attention with recent commercialization successes. A major impairment in FSO links is the turbulenceinduced fading which severely degrades the link performance. To mitigate turbulence-induced fading and, therefore, to improve the error rate performance, spatial diversity can be used over FSO links which involves the deployment of multiple laser transmitters/receivers. In this paper, we investigate the bit error rate (BER) performance of FSO links with spatial diversity over lognormal atmospheric turbulence fading channels, assuming both independent and correlated channels among transmitter/receiver apertures. Our analytical derivations build upon an approximation to the sum of correlated log-normal random variables. The derived BER expressions quantify the effect of spatial diversity and possible spatial correlations in a log-normal channel.
\end{abstract}

Index Terms-Atmospheric turbulence, bit error rate, error rate performance analysis, free space optical communication, lognormal channel, MIMO.

\section{INTRODUCTION}

$\mathbf{F}$ REE-SPACE optical (FSO) communications is a costeffective, license-free, and high bandwidth access technique, which has attracted significant attention recently for a variety of applications [1]-[4]. Despite the major advantages of FSO communications, its widespread use is hampered by several challenges in practical deployment. For example, aerosol scattering caused by rain, snow, and fog results in performance degradations, leaving the FSO link vulnerable to adverse weather conditions [5]. Another possible impairment over FSO links is building-sway as a result of wind loads, thermal expansion, and weak earthquakes [6], [7]. A major impairment is the effect of atmospheric turbulence [8], which will be the focus of this paper. Atmospheric turbulence occurs as a result of the variations in the refractive index due to inhomogeneties in temperature and pressure changes. This results in rapid fluctuations at the received signal, i.e., signal fading, impairing the link performance severely. Although

Manuscript received February 11, 2006; revised September 15, 2006 and January 26, 2007; accepted January 28, 2007. The associate editor coordinating the review of this letter and approving it for publication was X. Wang. This paper was presented in part at IEEE VTC'04-Fall, Los Angeles, California, USA, September 2004. The work of S. M. Navidpour and M. Kavehrad is supported in part by a DARPA Grant sponsored by the U.S. Air Force Research Laboratory/Wright-Patterson AFB Contract-FA8650-04C-7114 and The Pennsylvania State University CICTR. The work of M. Uysal is supported in part by an NSERC Special Opportunity Grant (SROPJ30582105).

S. M. Navidpour and M. Kavehrad are with the Department of Electrical Engineering, Pennsylvania State University, University Park, PA 16802 USA (e-mail: navidpour@psu.edu, mkavehrad@psu.edu).

M. Uysal is with the Department of Electrical and Computer Engineering, University of Waterloo, Waterloo, Ontario, Canada, N2L 3G1 (e-mail: muysal@ece.uwaterloo.ca).

Digital Object Identifier 10.1109/TWC.2007.06109.
FSO links are built taking into account a certain dynamic margin, the practical limitations on link budgets do not allow very high margins leaving the link vulnerable to deep fades. Powerful fading-mitigation techniques need to be deployed for FSO links particularly with transmission range of $1 \mathrm{~km}$ or longer. Error control coding in conjunction with interleaving can be employed in FSO communications to combat fading [9], [10]. However, optical links with their transmission rates of order of gigabits exhibit high temporal correlation. For most scenarios, this requires large-size interleavers to achieve the promised coding gains. Based on the statistical properties of turbulence-induced fading, maximum likelihood sequence detection (MLSD) is proposed in [11] as another solution for fading mitigation. However, MLSD requires complicated multidimensional integrations and suffers from excessive computational complexity. Some sub-optimal temporal-domain fading mitigation techniques are further explored in [11], [12].

Spatial diversity techniques ${ }^{1}$ [13], i.e., the employment of multiple transmit/receive apertures, provide an attractive alternative approach for fading compensation with their inherent redundancy. Besides its role as a fading-mitigation tool, multiple-aperture designs significantly reduce the potential for temporary blockage of the laser beam by obstructions (e.g., birds). Further justification for the employment of multiple apertures comes from limitations in transmit power density (expressed in terms of milliwatts per square centimeter). The allowable safe laser power depends on the wavelength and obviously a higher power at the receiver side allows the system to support longer distances and through heavier attenuation while achieving higher data rates. Information theoretic bounds for MIMO FSO links have been first studied in [14], where ergodic capacity and outage capacity are derived for intensitymodulation/direct-detection (IM/DD) FSO links operating in log-normal modeled atmospheric turbulence. Under the assumption of shot-noise-limited regime with Poisson statistics, it is demonstrated that ergodic capacity scales as the number of transmit apertures times the number of receive apertures for high signal-to-background noise ratio. Outage probability for MIMO FSO links are derived in [15] assuming Gaussian noise statistics that can be considered as a limiting case of Poisson statistics. We should also note an earlier experimental study in [16] where Kim et.al. measure the performance of a MIMO FSO link and discuss practical design issues such as transmitter spacing and spacing patterns, e.g., circular vs. rectangular.

\footnotetext{
${ }^{1}$ This paper does not consider space-time coding or multiplexing techniques. In the following, MIMO (multi-input multi-output) term is sometimes used to refer to the deployment of multiple-transmit and multiple-receive apertures.
} 
The standard performance metric adopted by most FSO manufacturers is the bit error rate (BER) [1]. Analytical BER performance for a single-receiver FSO link is derived in [17] while simulated BER performance results are demonstrated in [8] for a dual-receiver FSO link. Since the typical BER target is set as $10^{-9}$ for most practical applications, this brings a large computational time for Monte-Carlo type simulation experiments. Therefore, development of analytical tools for BER performance are helpful in providing extensive comparative analysis among different FSO configurations, which will be the main focus of this paper. In this paper, we derive BER expressions for FSO links with multiple transmit and/or receive apertures with and without channel state information (CSI) considering both spatially independent and correlated channels. The derived expressions quantify the effect of spatial diversity and spatial correlations in a log-normal channel.

The rest of the paper is organized as follows: In Section II, we introduce the system model and describe lognormal atmospheric turbulence channel under consideration. In Section III, we present BER expressions for FSO links with multiple transmitter and/or receiver apertures. In Section IV, we provide extensive numerical examples to confirm the accuracy of the derived expressions for various FSO configurations and discuss the effect of spatial diversity on the FSO link performance. Finally, conclusions are presented in Section V.

\section{SySTEM Model}

We consider a FSO link with $M$ transmit and $N$ receive apertures. We assume high signal-to-noise ratio (SNR) regime where we can use Gaussian noise model ${ }^{2}$ [8]. Assuming on-off keying (OOK), the received signal at the $n$th receive aperture is then given as

$$
r_{n}=s \eta \sum_{m=1}^{M} I_{m n}+v_{n}, n=1, \ldots, N
$$

where $s \in\{0,1\}$ is the transmitted information bit, $\eta$ is the optical-to-electrical conversion coefficient, and $\nu_{n}$ is additive white Gaussian noise with zero mean and variance of $\sigma_{v}^{2}=N_{0} / 2$. The fading channel coefficient which models the channel from the $m$ th transmit aperture to the $n$th receive aperture is given by

$$
I_{m n}=I_{0} \exp \left(2 X_{m n}\right)
$$

where $I_{0}$ is the signal light intensity without turbulence and $X_{m n}$ are identically (not necessarily independent) distributed normal random variables with mean $\mu_{x}$ and variance $\sigma_{x}^{2}$. Therefore, $I_{m n}$ follows a lognormal distribution

$$
f\left(I_{m n}\right)=\frac{1}{2 I_{m n}} \frac{1}{\sqrt{2 \pi \sigma_{x}^{2}}} \exp \left(-\frac{\left(\ln \left(I_{m n} / I_{0}\right)-2 \mu_{x}\right)^{2}}{8 \sigma_{x}^{2}}\right)
$$

To ensure that the fading does not attenuate or amplify the average power, we normalize the fading coefficients such that

\footnotetext{
${ }^{2}$ Precise characterization of practical photo-detectors requires a complex (and analytically intractable) statistical model including bandwidth limitations and mixtures of noise processes. However, considering the first and second moments of photo-detector outputs and employing central limit theorem, the filtered Poisson process at the detector output can be approximated as a Gaussian process with high accuracy.
}

$E\left[\left|I_{m n} / I_{0}\right|\right]=1$. Doing so requires the choice of $\mu_{x}=-\sigma_{x}^{2}$ [18]. Assuming weak turbulence conditions, the variances of log-amplitude fluctuation of plane and spherical waves are respectively given by [19]

$$
\begin{gathered}
\left.\sigma_{x}^{2}\right|_{\text {plane }}=0.307\left(\frac{2 \pi}{\lambda}\right)^{7 / 6} L^{11 / 6} C_{n}^{2} \\
\left.\sigma_{x}^{2}\right|_{\text {spherical }}=0.124\left(\frac{2 \pi}{\lambda}\right)^{7 / 6} L^{11 / 6} C_{n}^{2}
\end{gathered}
$$

where $\lambda$ is the wavelength and $L$ is the link distance in meters. $C_{n}^{2}$ stands for the refractive index structure coefficient and is altitude-dependent. Several $C_{n}^{2}$ profile models are available in the literature, but the most commonly used is the HufnagleValley model described by [2]

$$
\begin{array}{r}
C_{n}^{2}(h)=0.00594(v / 27)^{2}\left(10^{-5} h\right)^{10} \exp (h / 1000)+ \\
2.7 \times 10^{-6} \exp (-h / 1500)+A \exp (-h / 1000)
\end{array}
$$

where $h$ is the altitude in meters (m), $\nu$ is the rms wind speed in meters per second $(\mathrm{m} / \mathrm{sec})$, and $A$ is a nominal value of $C_{n}^{2}(0)$ at the ground in $m^{-2 / 3}$. For FSO links near the ground, $C_{n}^{2}$ can be taken approximately $1.7 \times 10^{-14} \mathrm{~m}^{-2 / 3}$ during daytime and $8.4 \times 10^{-15} \mathrm{~m}^{-2 / 3}$ at night. In general, $C_{n}^{2}$ varies from $10^{-13} \mathrm{~m}^{-2 / 3}$ for strong turbulence to $10^{-17} \mathrm{~m}^{-2 / 3}$ for weak turbulence with $10^{-15} \mathrm{~m}^{-2 / 3}$ often defined as a typical average value [20].

In this paper, we denote the correlation length and correlation time of intensity fluctuations as $d_{0}$ and $\tau_{0}$, respectively. Assuming $l_{0}<\sqrt{\lambda L}<L_{0}$, where $l_{0}$ and $L_{0}$ are inner and outer scales, $d_{0}$ can be approximated by $d_{0} \approx \sqrt{\lambda L}$ [8]. When the aperture size $D_{0}$ is much larger than the correlation length $d_{0}$, i.e., $D_{0}>>d_{0}$, the detrimental effect of turbulence-induced fading is reduced due to the aperture averaging [2]. However, it is not always possible to make the aperture large enough, which justifies the deployment of multiple photodetectors at the receiver side for scenarios with $D_{0}<d_{0}$. The spatial correlation matrix $\mathbf{R}$ to model the correlations among receive apertures is given as

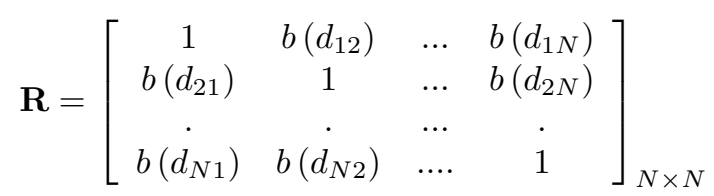

where $d_{i j}$ is the separation between the $i$ th and $j$ th receive apertures. In (6), $b(d)$ represents the normalized log-amplitude covariance function between two points in a receiving plane perpendicular to the direction of propagation and is defined by

$$
b\left(d_{P_{1}, P_{2}}\right)=\frac{E\left[X\left(P_{1}\right) X\left(P_{2}\right)\right]-E\left[X\left(P_{1}\right)\right] E\left[X\left(P_{2}\right)\right]}{\sigma_{x}^{2}}
$$

where $d_{P_{1}, P_{2}}$ is the distance between $P_{1}$ and $P_{2}$. Then the spatial covariance matrix $\boldsymbol{\Gamma}$ is given by $\boldsymbol{\Gamma}=\sigma_{x}^{2} \mathbf{R}$. Similarly, a correlation matrix of size $M \times M$ in the form of (6) and corresponding covariance matrix can be defined for modeling the spatial correlation at the transmitter side. 


\section{DERIVATION OF BER EXPRESSIONS}

In this section, we present BER expressions for FSO links with spatial diversity. First, we study the BER performance of a SISO (single-input single-output) FSO link that will be used as a benchmark for spatial diversity systems under consideration.

\section{A. SISO FSO Link}

Assuming OOK modulation and perfect CSI available at the receiver side, the bit error rate is calculated as

$$
P_{e}=p(\text { off }) p(\mathrm{e} \mid \mathrm{off})+p(\text { on }) p(\mathrm{e} \mid \mathrm{on})
$$

where $p$ (on) and $p$ (off) are the probabilities of transmitting "on" and "off" bits, respectively. $p$ (e|off) and $p(\mathrm{e} \mid \mathrm{on})$ denote the conditional bit error probabilities when the transmitted bit is "off" or "on". Conditioned on the fading coefficient $I^{3}$, we have

$$
\begin{aligned}
& p(e \mid \text { off }, I)=p(\text { e } \mid \text { on, } I)= \\
& p\left(v>\frac{\eta I}{2}\right)=p\left(v<-\frac{\eta I}{2}\right)=Q\left(\frac{\eta I}{\sqrt{2 N_{0}}}\right)
\end{aligned}
$$

Averaging over the fading coefficient, we obtain

$$
p(\mathrm{e} \mid \mathrm{off})=p(\mathrm{e} \mid \mathrm{on})=\int_{0}^{\infty} f_{I}(I) Q\left(\frac{\eta I}{\sqrt{2 N_{0}}}\right) d I
$$

where $Q($.$) is the Gaussian-Q function defined as Q(y)=$ $(1 / \sqrt{2 \pi}) \int_{y}^{\infty} \exp \left(-t^{2} / 2\right) d t$. Considering the symmetry of the problem, i.e., $p(\mathrm{on})=p(\mathrm{off})=1 / 2$ and $p(e \mid \mathrm{on})=$ $p(e \mid$ off $)$ and replacing $I$ in terms of $x, P_{e}$ can be obtained as

$$
\begin{aligned}
P_{e} & =\int_{0}^{\infty} f_{I}(I) Q\left(\frac{\eta I}{\sqrt{2 N_{0}}}\right) d I \\
& =\int_{-\infty}^{\infty} \Omega\left(x,-\sigma_{x}^{2}, \sigma_{x}^{2}\right) Q\left(\frac{\eta I_{0} e^{2 x}}{\sqrt{2 N_{0}}}\right) d x
\end{aligned}
$$

where $\Omega(u, v, w)$ is defined by $\Omega(u, v, w)=$ $(1 / \sqrt{2 \pi w}) \exp \left(-(u-v)^{2} / 2 w\right)$. The integration in (11) can be efficiently computed by Gauss-Hermite quadrature formula [21]

$$
P_{e} \approx \frac{1}{\sqrt{\pi}} \sum_{i=1}^{k} w_{i} Q\left(\frac{\eta I_{0} e^{-2 \sigma_{x}^{2}+z_{i} \sqrt{8 \sigma_{x}^{2}}}}{\sqrt{2 N_{0}}}\right)
$$

where $k$ is the order of approximation, $z_{i}, i=1, \ldots, k$ are the zeros of the $k$ th-order Hermite polynomial, and $w_{i}, i=$ $1, \ldots, k$ are weight factors for the $k$ th-order approximation.

So far, we have assumed the availability of perfect CSI. Now, we assume that the receiving part has knowledge of the distribution of the channel fades, but has no knowledge of the instantaneous fading state. In this case, the maximum likelihood (ML) detection is implemented by thresholding the received signal based on the likelihood function. The conditional bit error probabilities are given by

$$
p(\mathrm{e} \mid \mathrm{on})=\int_{\Lambda(r)<1} \operatorname{Pr}(r \mid \mathrm{on}) d r
$$

\footnotetext{
${ }^{3}$ Since SISO link is considered, indexes $m$ and $n$ in (1) are dropped.
}

$$
p(\mathrm{e} \mid \mathrm{off})=\int_{\Lambda(r)>1} \operatorname{Pr}(r \mid \mathrm{off}) d r
$$

where the decision regions are determined based on the likelihood function defined as [8]

$$
\begin{aligned}
\Lambda(r) & =\frac{p(r \mid \mathrm{on})}{p(r \mid \mathrm{off})} \\
& =\int_{-\infty}^{\infty} \Omega\left(x,-\sigma_{x}^{2}, \sigma_{x}^{2}\right) \exp \left(-\frac{\left(r-\eta I_{0} e^{2 x}\right)^{2}-r^{2}}{N_{0}}\right) d x
\end{aligned}
$$

\section{B. FSO Link With Spatial Diversity}

Now, we focus our attention on FSO links with spatial diversity. Assuming both transmit and receive diversity are employed, the optimum decision metric for on-off keying is given by

$$
p\left(\mathbf{r} \mid \text { on }, I_{m n}\right) \underset{\text { off }}{\stackrel{\text { on }}{\gtrless}} p\left(\mathbf{r} \mid \text { off }, I_{m n}\right)
$$

where $\mathbf{r}=\left(r_{1}, r_{2}, \ldots, r_{N}\right)$ is the received signal vector and the conditional probabilities are

$$
\begin{gathered}
p\left(\mathbf{r} \mid \text { on }, I_{m n}\right)=\frac{\exp \left(-\frac{1}{2 \sigma_{v}^{2}} \sum_{n=1}^{N} r_{n}^{2}\right)}{\left(2 \pi \sigma_{v}^{2}\right)^{\frac{N}{2}}} \\
p\left(\mathbf{r} \mid \text { off }, I_{m n}\right)=\frac{\exp \left(-\frac{1}{2 \sigma_{v}^{2}} \sum_{n=1}^{N}\left(r_{n}-\eta \sum_{m=1}^{M} I_{m n}\right)^{2}\right)}{\left(2 \pi \sigma_{v}^{2}\right)^{\frac{N}{2}}}
\end{gathered}
$$

for "on" and "off" states, respectively. Replacing (17), (18) in (16) and dropping out common terms which do not affect the decision process, we have

$$
-\sum_{n=1}^{N}\left(r_{n}-\eta \sum_{m=1}^{M} I_{m n}\right)^{2} \stackrel{\text { on }}{\gtrless}-\sum_{\text {off }}^{N} r_{n}^{2}
$$

which can be further simplified as

$$
\sum_{n=1}^{N} \sum_{m=1}^{M} \eta I_{m n} r_{n} \underset{\text { off }}{\gtrless} \frac{\text { on }}{2} \sum_{n=1}^{N}\left(\eta \sum_{m=1}^{M} I_{m n}\right)^{2} .
$$

The conditional bit error probabilities are given as in (21) and (22) at the top of the next page. Noting $p_{e}\left(\right.$ off $\left.\mid I_{m n}\right)=$ $p_{e}\left(\right.$ on $\left.\mid I_{m n}\right)$ and averaging over the fading coefficients, we obtain $P_{e}$ as

$$
P_{e}=\int_{\mathbf{x}} f_{\mathbf{x}}(\mathbf{x}) Q\left(\frac{\eta I_{0}}{2 M N \sigma_{v}} \sqrt{\sum_{n=1}^{N}\left(\sum_{m=1}^{M} e^{2 x_{m n}}\right)^{2}}\right) d \mathbf{x}
$$

where $f_{\mathbf{x}}(\mathbf{x})$ is the joint probability density function (pdf) of Gaussian vector $\mathbf{x}=\left(x_{11}, x_{12} \ldots, x_{N M}\right)$ of length $M N$. One should note that the scaling factor $M N$ appears in the argument of $Q($.$) function in (23). The factor M$ is included to ensure that the total power of diversity system is the same as the power of the benchmark SISO link, i.e., no diversity system, for the sake of fair comparison. The factor $N$, on the other hand, ensures that the sum of the $N$ receive aperture areas is the same as the area of the receive aperture of the SISO 


$$
\begin{aligned}
& p_{e}\left(\text { off } \mid I_{m n}\right)=p\left(\sum_{n=1}^{N} \sum_{m=1}^{M} \eta I_{m n} r_{n}>\frac{1}{2} \sum_{n=1}^{N}\left(\eta \sum_{m=1}^{M} I_{m n}\right)^{2} \mid r_{n}=v_{n}\right) \\
& =p\left(\sum_{n=1}^{N} \sum_{m=1}^{M} \eta I_{m n} v_{n}>\frac{1}{2} \sum_{n=1}^{N}\left(\eta \sum_{m=1}^{M} I_{m n}\right)^{2}\right)=Q\left(\frac{1}{2 \sigma_{v}} \sqrt{\sum_{n=1}^{N}\left(\eta \sum_{m=1}^{M} I_{m n}\right)^{2}}\right)
\end{aligned}
$$

$$
\begin{aligned}
& p_{e}\left(\text { on } \mid I_{m n}\right)=p\left(2 \sum_{n=1}^{N} \sum_{m=1}^{M} \eta I_{m n} r_{n}<\sum_{n=1}^{N}\left(\eta \sum_{m=1}^{M} I_{m n}\right)^{2} \mid r_{n}=\eta \sum_{m=1}^{M} I_{m n}+v_{n}\right) \\
& =p\left(2 \sum_{n=1}^{N} \sum_{m=1}^{M} \eta I_{m n}\left(\eta \sum_{m=1}^{M} I_{m n}+v_{n}\right)<\sum_{n=1}^{N}\left(\eta \sum_{m=1}^{M} I_{m n}\right)^{2}\right)=Q\left(\frac{1}{2 \sigma_{v}} \sqrt{\sum_{n=1}^{N}\left(\eta \sum_{m=1}^{M} I_{m n}\right)^{2}}\right)
\end{aligned}
$$

$\operatorname{link}^{4}$ [15]. Although (23) can be calculated through numerical multi-dimensional integration, it does not yield a closed-form solution. To have further insight into the performance, we consider transmit diversity and receive diversity separately in the following.

1) Transmit Diversity: Replacing $N=1$ in (23), we have

$$
P_{e}=\int_{\mathbf{x}} f_{\mathbf{x}}(\mathbf{x}) Q\left(\frac{\eta I_{0}}{2 M \sigma_{v}} \sum_{m=1}^{M} e^{2 x_{m}}\right) d \mathbf{x}
$$

which requires the solution of an $M$-dimensional integration. Approximating the sum of log-normal random variables as $\log$-normal, i.e., $e^{2 x} \approx \sum_{m=1}^{M} e^{2 x_{m}}$ (See Appendix), (24) reduces to a one-dimensional integration given by

$$
P_{e} \approx \int_{-\infty}^{\infty} \Omega\left(x,-\hat{\sigma}_{x}^{2}, \hat{\sigma}_{x}^{2}\right) Q\left(\frac{\eta I_{0} e^{2 x}}{\sqrt{2 N_{0}}}\right) d x
$$

where we define the effective variance $\hat{\sigma}_{x}^{2}=\sigma_{x}^{2} / M$. It is interesting to note that this expression has the same form as (11) which has been obtained for the SISO link with a scaled variance. In other words, the underlying multi-input singleoutput (MISO) channel can be represented by an equivalent SISO channel with appropriate scaling in the channel variance. It should be further emphasized that our result confirms the earlier observations reported in [16] obtained through an experimental study. It is argued in [16] that linear scaling does not hold anymore for larger link distances and intuitively discussed that correlation among transmitting paths reduces the achievable maximum diversity order. This effect can be readily observed from our expressions in a precise manner. Specifically, we observe from the appendix that correlation manifests itself in the form of an increase in the effective lognormal variance $\hat{\sigma}_{x}^{2}$, i.e.,

$$
\hat{\sigma}_{x}^{2}=\frac{\sigma_{x}^{2}}{M}+\frac{1}{M^{2}} \sum_{\substack{k=1 \\ k \neq l}}^{M} \Gamma_{k l}
$$

\footnotetext{
${ }^{4}$ In radio frequency (RF) wireless communication links with omnidirectional antenna characteristics, deployment of multiple receive antennas increases the overall average signal-to-noise ratio at the receiver side resulting in so-called "array gain". However, in FSO communication, deployment of multiple receive apertures does not necessarily guarantee increase in the received power. Since FSO relies line-of-sight propagation, the transmitter beam needs to be chosen more divergent to reach to the multiple receive apertures. However, beam divergence effectively reduces the received power over a fixed receive aperture area.
}

where $\Gamma_{k l}, l \neq k, l, k=1, \ldots, M$ are the correlation coefficients.

Under the assumption that instantaneous CSI is not available at the receiver side, the likelihood function is given by

$$
\Lambda(r)=\int_{\mathbf{x}} f_{\mathbf{x}}(\mathbf{x}) \exp \left[-\frac{\left(r-\frac{\eta I_{0}}{M} \sum_{m=1}^{M} e^{2 x_{m}}\right)^{2}-r^{2}}{N_{0}}\right] d \mathbf{x}
$$

Since the computation of (27) is required to determine the optimal decision regions for this case, the complexity of detection process is higher due to the involved multidimensional integrations. However, it is possible to simplify (27) by using the log-normal approximation which yields the following onedimensional integration

$$
\Lambda(r)=\int_{-\infty}^{\infty} \Omega\left(x, \frac{-\sigma_{x}^{2}}{M}, \frac{\sigma_{x}^{2}}{M}\right) \exp \left(-\frac{\left(r-\eta I_{0} e^{2 x}\right)^{2}-r^{2}}{N_{0}}\right) d x
$$

Noting the similarity of (28) and (15), we conclude that our observation of MISO channel representation in terms of equivalent SISO channel with scaled variance remains valid even in the lack of CSI at the receive side.

2) Receive Diversity: First we assume optimal combining with perfect CSI. Replacing $M=1$ in (23), we have 5

$$
P_{e}=\int_{\mathbf{x}} f_{\mathbf{x}}(\mathbf{x}) Q\left(\frac{\eta I_{0}}{2 N \sigma_{v}} \sqrt{\sum_{n=1}^{N} e^{4 x_{n}}}\right) d \mathbf{x}
$$

which requires the solution of a $N$-dimensional integration. Applying log-normal approximation to $e^{x} \approx \sum_{n=1}^{N} e^{4 x_{n}},(29)$ reduces to a one-dimensional integral given by

$$
P_{e} \approx \int_{-\infty}^{+\infty} \Omega\left(x, \hat{\mu}, \hat{\sigma}_{x}^{2}\right) Q\left(\frac{\eta I_{0}}{2 N \sigma_{v}} e^{x / 2}\right) d x
$$

where the effective variance and mean are defined by $\hat{\sigma}_{x}^{2}=$ $\left(1 / N^{2}\right) \sum_{l, k} 16 \Gamma_{k l}$ and $\hat{\mu}=\log N+4 \sigma_{x}^{2}-\hat{\sigma}_{x}^{2} / 2$.

\footnotetext{
${ }^{5}$ In this scheme, the sum of the $N$ receive aperture areas is the same as the area of the receive aperture of the SISO link. Therefore, considering background noise limited receivers, the variance of the noise is given by $\sigma_{\nu}^{2}=N_{0} / 2 N[15]$
} 
As earlier noted, a possible alternative in the implementation of receive diversity for FSO links is to exploit the inherent aperture averaging; that can be readily obtained by deploying single receive aperture with a large collecting area instead of the deployment of several apertures with smaller collecting areas. To let a performance comparison between aperture averaging and receive diversity through multiple apertures, we consider the following decision rule

$$
r=\sum_{n=1}^{N} r_{n} \gtrless \frac{\eta}{2} \sum_{n=1}^{N} I_{n} .
$$

We call this receiver "equal gain combining" (EGC) with a slight abuse of term ${ }^{6} . P_{e}$ expression is obtained as

$$
P_{e}=\int_{\mathbf{x}} f_{\mathbf{x}}(\mathbf{x}) Q\left(\frac{\eta I_{0}}{2 N \sigma_{v}} \sum_{n=1}^{N} e^{2 x_{n}}\right) d \mathbf{x} .
$$

Based on our log-normal approximation, (32) simplifies to (25) where the scaled variance is now given by $\hat{\sigma}_{x}^{2}=\sigma_{x}^{2} / N$. Assuming the further deployment of transmit diversity, it can be easily shown that the effective log-normal variance will be reduced to $\sigma_{x}^{2} / M N$.

\section{NumericAl RESUlts}

In this section, we present numerical results for the BER performance of FSO links for various numbers of transmit/receive apertures and correlation values. We consider a FSO system with a receive aperture of size $D_{0}=5 \mathrm{~cm}$ and the wavelength of $\lambda=1.55 \mu \mathrm{m}$. The link distance is assumed to be $L=2 \mathrm{~km}$. The correlation length can be therefore approximated as $d_{0} \approx \sqrt{\lambda L}=5.5 \mathrm{~cm}$.

Fig. 1 illustrates the BER performance of a FSO link with $M=2$ and 3 transmit apertures over a turbulence channel with standard deviation of $\sigma_{x}=0.1$ and $\sigma_{x}=0.3$. We present both the exact expressions and their approximations obtained through (24) and (25), respectively. It is observed that our approximate expressions which are formulated in terms of a single integral give excellent matches to the exact expressions which require multidimensional integrations. The difference is negligible for the considered values of log-normal variance. The performance of SISO FSO link is also included in Fig. 1 as a benchmark. As the figure clearly illustrates, the performance improves significantly with the increasing number of transmit apertures which, in effect, reduces the effective log-normal variance of the diversity channel. Specifically, the transmit diversity reduces log-normal variance by a factor of $M$. For example, the performance of 2-TX and 3-TX systems operating over a log-normal channel with a variance of $\sigma_{x}^{2}=(0.3)^{2}=0.09$ can be well-approximated through (25) by equivalent 1-TX systems over a log-normal channel with variances of $\sigma_{x}^{2} / 2=(0.3)^{2} / 2=0.045$ and $\sigma_{x}^{2} / 3=(0.3)^{2} / 3=0.03$ (c.f. lines labeled as "2-TX approx" and "3-TX approx.")

In Fig. 2, we demonstrate the effect of spatial correlation on the FSO link performance. We consider a FSO link with

\footnotetext{
${ }^{6} \mathrm{~A}$ similar scheme in [8] is called EGC where the receiver outputs are directly combined. The receiver in [8] does not require instantaneous CSI. In our decision rule, we still assume the availability of CSI for threshold calculation.
}

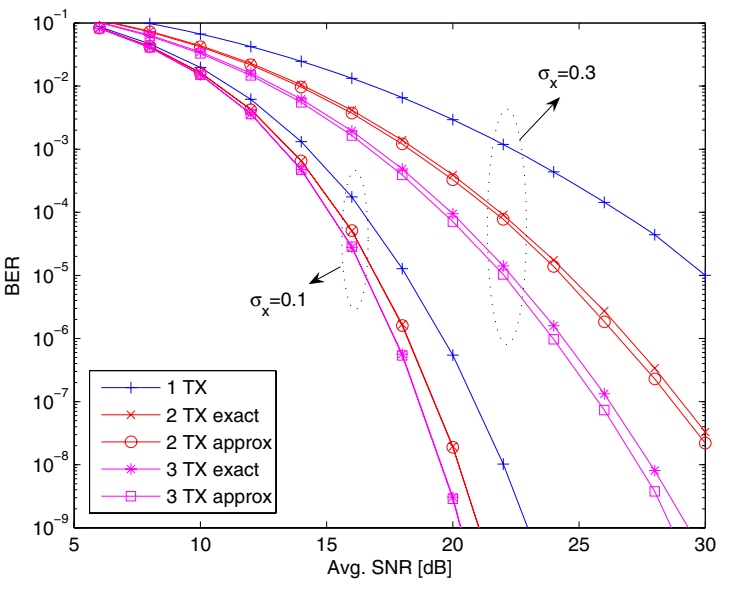

Fig. 1. Comparison of exact and approximate BER expressions for FSO links with two and three transmit apertures assuming perfect CSI.

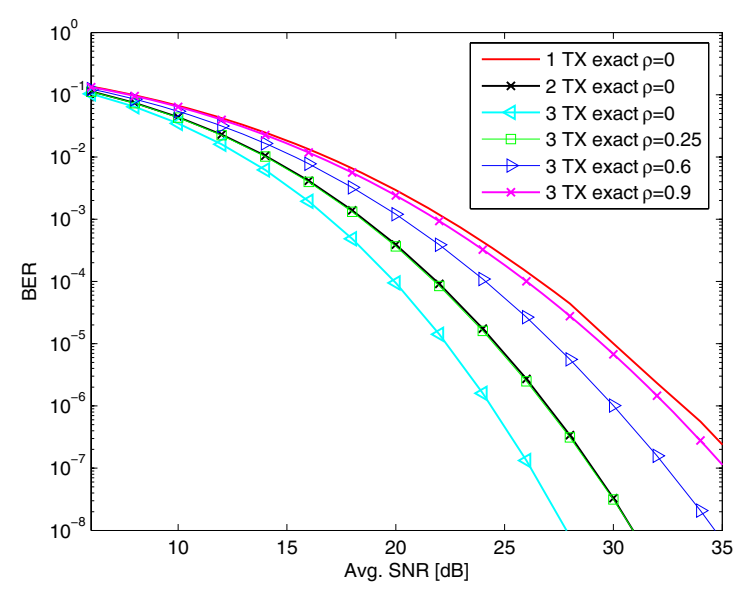

Fig. 2. Effect of spatial correlation on the performance of a FSO link with three transmit apertures over a log-normal channel with $\sigma_{x}=0.3$. Performance curves for one and two transmit apertures over spatially independent channels $(\rho=0)$ are included as benchmarks.

$M=3$ transmit apertures which are equidistant from each other and assume correlation values of $b(d)=\rho=0.25,0.6$, and 0.9 where $d$ is the distance between the apertures. From comparison to the case of spatially independent channels, it can be clearly observed that even a correlation value of $\rho=$ 0.25 among 3 transmit apertures degrades the performance significantly, decreasing the diversity order by one, i.e., it achieves a similar performance expected for spatially independent dual transmit apertures. As the correlation increases, the performance loss is observed to be much more severe. For example, the performance for the correlation value of $\rho=0.9$ comes very close to that for the single transmit aperture. These observations are also in contrast to what we typically see in RF wireless communications, where only the full spatial correlation results in the loss of diversity order [23]. Our observations demonstrate that efficient separation between apertures is crucial to achieve the promised diversity gains from multiple transmitters. It should be further noted that our approximation approach works fine for the correlated case as well and comparison with exact results are omitted 


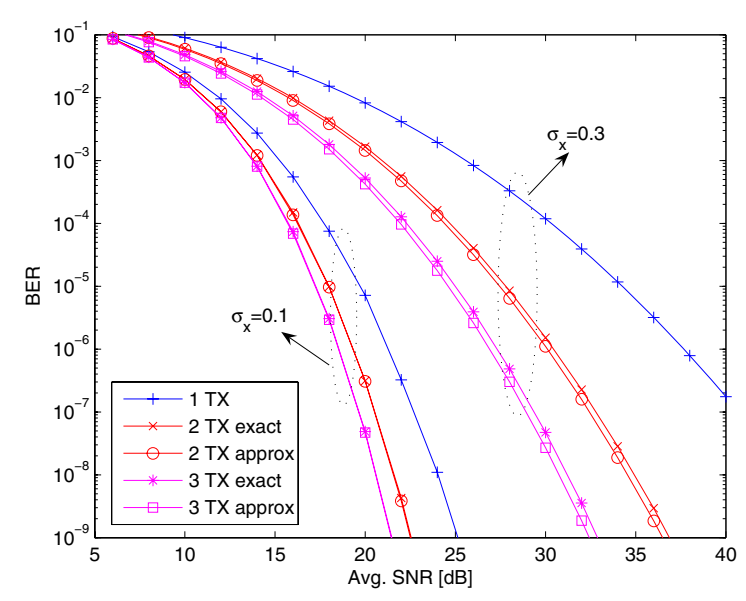

Fig. 3. Comparison of exact and approximate BER expressions for FSO links with two and three transmit apertures assuming no instantaneous CSI.

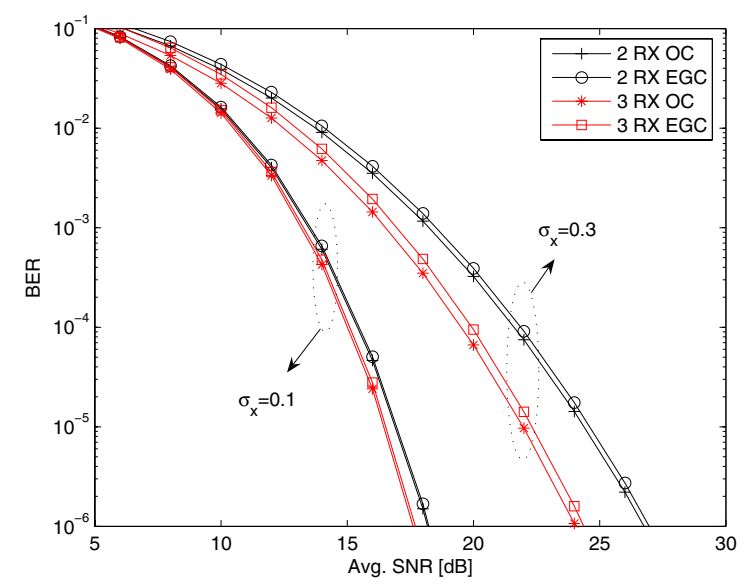

Fig. 4. Comparison of OC and EGC receivers for a FSO link with two and three receive apertures.

here for the sake of brevity.

In Fig. 3, we assume no instantaneous CSI is available and present the BER performance of a FSO link with $M=1$, 2 and 3 transmit apertures. The exact expressions in this figure are obtained through the exact threshold of (27) which require multi-dimensional integrals. The approximations are given by (28) and require only single-dimension integrals and demonstrate a perfect match to the exact ones. Similar to Fig. 1, we observe performance improvement with increasing number of transmit apertures. Although the general behavior of plots is similar to those of Fig. 1, a performance loss is observed in comparison to perfect CSI case for various values of $\sigma_{x}$ ranging from $1.5 \mathrm{~dB}$ to $4.5 \mathrm{~dB}$ at $\mathrm{BER}=10^{-5}$.

Fig. 4 compares the performance of EGC and OC receivers for FSO links with $N=2$ and 3 receive apertures. It is observed that the performance of EGC receiver is very close to that of the optimal receiver. Specifically, we observe that there is only a $0.3 \mathrm{~dB}$ difference at $\mathrm{BER}=10^{-5}$ for $\sigma_{x}=0.3$. This difference further decreases for lower values of log-normal variance. The observation that performances of OC and EGC receivers are similar also demonstrates that the receive diversity can be obtained in practice through aperture averaging

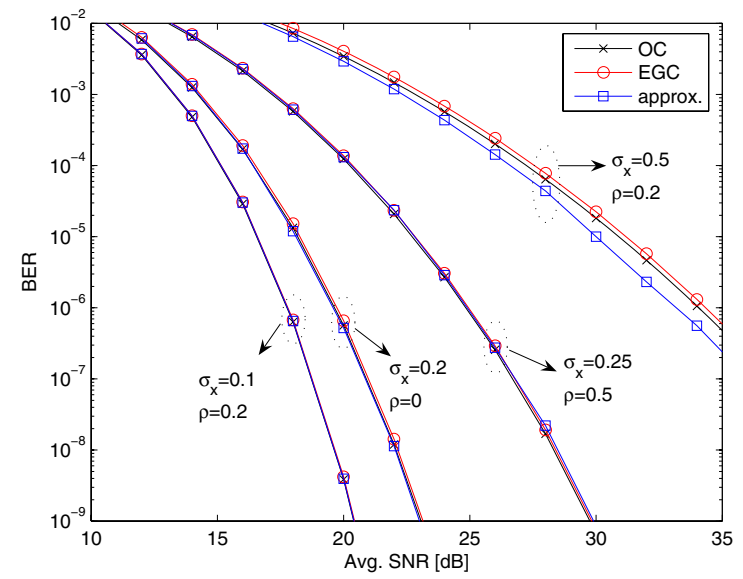

Fig. 5. BER performance of a FSO link with two transmit and two receive apertures over spatially correlated channels.

effect, i.e., the deployment of a large receive aperture will provide a similar performance to that can be obtained by several separate smaller receive apertures.

Fig. 5 illustrates the BER performance of a MIMO FSO link with 2 transmit and 2 receive apertures. We assume identical correlation values for both the transmitter and receiver sides, i.e., $\rho_{R}=\rho_{T}=\rho$. The spatial correlation matrix of the MIMO FSO channel is modeled by the Kronecker product of the spatial correlation matrices of the transmitter and the receiver, i.e. $\mathbf{R}_{\text {MIMO }}=\mathbf{R}_{\mathbf{T}} \otimes \mathbf{R}_{\mathbf{R}}$. We present exact BER performance curves of $\mathrm{OC}$ and EGC implementations for various log-normal variance and correlation values. $\mathrm{OC}$ and EGC present very similar performances demonstrating that EGC can be used as a reliable and simpler alternative to OC for most practical purposes in a MIMO setting. Fig. 5 further illustrates the accuracy of our log-normal approximation. It is observed that the approximation, c.f., (32), provides an excellent match to the exact performance for channel conditions with weak turbulence and low correlation. Although there is some discrepancy observed for higher log-normal variance, the approximation still captures the characteristic behaviour of the exact performance curves.

\section{CONCLUSION}

In this paper, we have investigated the BER performance of FSO links over log-normal atmospheric turbulence channels with spatial diversity. Our results demonstrate that FSO links with transmit and receive diversity can be efficiently represented by equivalent SISO systems with appropriate scaling in the channel variance. In other words, the effect of spatial diversity manifests itself as a decrease of the channel variance. We also observe that the performance loss due to spatial correlation might be severe, demonstrating that efficient separation between apertures and strict co-alignment is crucial to achieve the promised diversity gains from multiple transmitters/receivers.

\section{APPENDIX}

This appendix provides an approximation for the summation of correlated log-normal random variables. Specifically, 
we want to approximate $\sum_{k=1}^{K} \exp \left(u_{k}\right)$ with a single lognormal random variable $e^{z}$ where $z$ is a Gaussian random variable with a mean of $\mu_{z}$ and a variance of $\sigma_{z}^{2}$. Defining $\operatorname{cov}\left(u_{k}, u_{l}\right)=\nu_{k l}, E\left(u_{k}\right)=\mu_{k}$ and using the results reported in [15] and [24], we have

$$
\begin{gathered}
\mu_{z}=\log \left(\alpha / \sqrt{1+\beta^{2} / \alpha^{2}}\right), \\
\sigma_{z}^{2}=\log \left(1+\beta^{2} / \alpha^{2}\right) .
\end{gathered}
$$

Here, $\alpha$ and $\beta$ are defined as

$$
\begin{gathered}
\alpha=\sum_{k=1}^{K} e^{\mu_{k}+\nu_{k k} / 2} \\
\beta^{2}=\sum_{k=1}^{K} \sum_{l=1}^{K} e^{\mu_{k}+\mu_{l}+\frac{\left(\nu_{k k}+\nu_{l l}\right)}{2}}\left(e^{v_{k l}}-1\right)
\end{gathered}
$$

respectively. Note that, due to power normalization (i.e., $\left.E\left[I / I_{0}\right]=E\left[\exp \left(2 x_{k}\right)\right]=E\left[\exp \left(u_{k}\right)\right]=1\right)$, we have $E\left(x_{k}\right)=-\sigma_{x}^{2}$ and $\mu_{k}=-\nu_{k k} / 2$. Further, under the assumption of weak turbulence (i.e,. $v_{k k}<<1$ ), and small correlation values ( i.e., $v_{k l}<<1$ ), (33) and (34) reduce to

$$
\begin{gathered}
\mu_{z}=\log K-\frac{\sigma_{z}^{2}}{2}, \\
\sigma_{z}^{2} \approx \frac{1}{K^{2}} \sum_{k, l} e^{v_{k l}}-1 \approx \frac{1}{K} v_{11}+\frac{1}{K^{2}} \sum_{k \neq l} v_{k l}
\end{gathered}
$$

where we assume that log-normal parameters are equal for all channels, i.e., $v_{k k}=v_{11}=4 \sigma_{x}^{2}$.

\section{ACKNOWLEDGMENT}

The authors would like to thanks the anonymous reviewers whose comments significantly improved the presentation of the paper. The second author thanks Dr. Jing Li for helpful discussions on an earlier draft version of this paper and $\mathrm{Mr}$. Majid Safari for his diligent work in the revision process.

\section{REFERENCES}

[1] H. Willebrand and B. S. Ghuman, Free Space Optics: Enabling Optical Connectivity in Today's Networks. Indianapolis, IN: Sams Publishing, 2002.

[2] L. C. Andrews, R. L. Phillips, and C. Y. Hopen, Laser Beam Scintillation with Applications. Bellingham, WA: SPIE Press, 2001.

[3] D. Kedar and S. Arnon, "Urban optical wireless communication networks: The main challenges and possible solutions," IEEE Commun. Mag., vol. 42, no. 5, pp. 2-7, May 2004.
[4] Wireless Communications Association International [Online]. Available: http://www.wcai.com/

[5] B. R. Strickland, M. J. Lavan, E. Woodbridge, and V. Chan "Effects of fog on the bit-error rate of a free space laser communication system," Applied Optics, vol. 38, no. 3, pp. 424-431, Jan. 1999.

[6] S. Arnon, "Optimization of urban optical wireless communication systems," IEEE Trans. Wireless Commun., vol. 2, no. 4, pp. 626-629, July 2003.

[7] S. Arnon, "Effects of atmospheric turbulence and building sway on optical wireless communication systems," Optics Lett., vol. 28, no. 2 , pp. 129-131, Jan. 2003.

[8] X. Zhu and J. M. Kahn, "Free-space optical communication through atmospheric turbulence channels," IEEE Trans. Commun., vol. 50, no. 8, pp. 1293-1300, Aug. 2002.

[9] X. Zhu and J. M. Kahn, "Performance bounds for coded free-space optical communications through atmospheric turbulence channels," IEEE Trans. Commun., vol. 51, no. 8, pp. 1233-1239, Aug. 2003.

[10] M. Uysal, S. M. Navidpour, and J. Li, "Error rate performance of coded free-space optical links over strong turbulence channels," IEEE Commun. Lett., vol. 8, no. 10, pp. 635-637, Oct. 2004.

[11] X. Zhu and J. M. Kahn, "Markov chain model in maximum-likelihood sequence detection for free-space optical communication through atmospheric turbulence channels," IEEE Trans. Commun., vol. 51, no. 3, pp. 509-516, Mar. 2003.

[12] X. Zhu, J. M. Kahn, and W. Jin, "Mitigation of turbulence-induced scintillation noise in free-space optical links using temporal-domain detection techniques," IEEE Photon. Technol. Lett., vol. 15, no. 4, pp. 623-625, Apr. 2003.

[13] A. Paulraj, R. Nabar, and D. Gore, Introduction to Space-Time Wireless Communications. Cambridge, UK: Cambridge University Press, 2003.

[14] S. M. Haas and J. H. Shapiro, "Capacity of wireless optical communications," IEEE J. Select. Areas Commun., vol. 21, no. 8, pp. 1346-1357, Oct. 2003.

[15] E. J. Shin and V. W. S. Chan, "Optical communication over the turbulent atmospheric channel using spatial diversity," IEEE GLOBECOM, Nov. 2002, pp. 2055-2060.

[16] I. Kim, H. Hakakha, P. Adhikari, and E. J. Korevaar, "Scintillation reduction using multiple transmitters," in Proc. SPIE, Feb. 1997, vol. 2990.

[17] C. C. Davis and I. Smolyaninov, "The effect of atmospheric turbulence on bit-error-rate in an on-off keyed optical wireless system," in Proc. SPIE Free-Space Laser Communication Laser Imaging, Mar. 1997, vol. 4489, pp. 126-137.

[18] S. M. Haas, Capacity of and coding for multiple-aperture wireless optical communications, Ph.D. dissertation, Massachusetts Institute of Technology, 2003.

[19] S. Karp, R. Gagliardi, S. E. Moran, and L. B. Stotts, Optical Channels. New York: Plenum, 1988.

[20] J. W. Goodman, Statistical Optics. New York: John Wiley \& Sons, 1985.

[21] M. K. Simon and M.-S. Alouini, Digital Communication over Fading Channels. New York: John Wiley \& Sons, 2000.

[22] J. G. Proakis, Digital Communications, 3rd ed. New York: McGrawHill, 1995.

[23] M. Uysal and C. N. Georgiades, "On the error performance analysis of space-time trellis codes," IEEE Trans. Wireless Commun., vol. 3, no. 4, pp. 1128-1133, July 2004.

[24] S. C. Schwartz and Y. S. Yeh, "On the distribution function and moments of power sums with log-normal components," Bell Syst. Tech. J., vol. 61, no. 7, pp. 1441-1462, Sep. 1982. 